Article

\title{
Entrance Pupil Irradiance Estimating Model for a Moon-Based Earth Radiation Observatory Instrument
}

\author{
Wentao Duan ${ }^{1}$, Shaopeng Huang ${ }^{2,3, *}$ and Chenwei Nie ${ }^{4}$ \\ 1 School of Human Settlements and Civil Engineering, Xi'an Jiaotong University, Xi'an 710054, China; \\ eilen.4011@stu.xjtu.edu.cn \\ 2 Institute of Deep Earth Science and Green Energy, Shenzhen University, Shenzhen 518060, China \\ 3 Department of Earth and Environmental Sciences, University of Michigan, Ann Arbor, MI 48109, USA \\ 4 Key Laboratory of Digital Earth Science, Institute of Remote Sensing and Digital Earth, Chinese Academy of \\ Sciences, Beijing 100094, China; niecw@radi.ac.cn \\ * Correspondence: shaopeng@szu.edu.cn
}

Received: 26 January 2019; Accepted: 6 March 2019; Published: 10 March 2019

\begin{abstract}
A Moon-based Earth radiation observatory (MERO) could provide a longer-term continuous measurement of radiation exiting the Earth system compared to current satellite-based observatories. In order to parameterize the detector for such a newly-proposed MERO, the evaluation of the instrument's entrance pupil irradiance (EPI) is of great importance. The motivation of this work is to build an EPI estimating model for a simplified single-pixel MERO instrument. The rationale of this model is to sum the contributions of every location in the MERO-viewed region on the Earth's top of atmosphere (TOA) to the MERO sensor's EPI, taking into account the anisotropy in the longwave radiance at the Earth TOA. Such anisotropy could be characterized by the TOA anisotropic factors, which can be derived from the Clouds and the Earth's Radiant Energy System (CERES) angular distribution models (ADMs). As an application, we estimated the shortwave (SW) $(0.3-5 \mu \mathrm{m})$ and longwave (LW) (5-200 $\mu \mathrm{m})$ EPIs for a hypothetic MERO instrument located at the Apollo 15 landing site. Results show that the SW EPI varied from 0 to $0.065 \mathrm{~W} / \mathrm{m}^{2}$, while the LW EPI ranged between 0.061 and $0.075 \mathrm{~W} / \mathrm{m}^{2}$ from 1 to 29 October, 2017. We also utilized this model to predict the SW and LW EPIs for any given location within the MERO-deployable region (region of $80.5^{\circ} \mathrm{W}-80.5^{\circ} \mathrm{E}$ and $81.5^{\circ} \mathrm{S}-81.5^{\circ} \mathrm{N}$ on the nearside of the Moon) for the future 18.6 years from October 2017 to June 2036. Results suggest that the SW EPI will vary between 0 and $0.118 \mathrm{~W} / \mathrm{m}^{2}$, while the LW EPI will range from 0.056 to $0.081 \mathrm{~W} / \mathrm{m}^{2}$. Though the EPI estimating model in this study was built for a simplistic single-pixel instrument, it could eventually be extended and improved in order to estimate the EPI for a multi-pixel MERO sensor.
\end{abstract}

Keywords: Earth radiation; MERO; entrance pupil irradiance; CERES; ADMs; TOA anisotropy

\section{Introduction}

The balance between the incoming solar radiation and the outgoing radiation is the driving force of the Earth's climate system. The incoming radiation mainly comes from solar radiation, while the outgoing radiation takes two forms: reflected solar shortwave (SW) radiation and longwave infrared (LW) radiation. Spaceborne platforms can provide global radiation observations, which are crucially needed for the Earth Radiation Budget (ERB) study. Thus, ERB research deeply relies on the development of space technology [1,2]. Since the early 1970s, several missions dedicated to the observation of Earth's outgoing radiations have been launched. The Earth Radiation Budget Experiment (ERBE) wide-field-of-view (WFOV) radiometer onboard the Earth Radiation Budget Satellite (ERBS) provided a 15-year (1984-1999) high-quality record of the Earth's radiant energy. It mainly measured the flux of SW and LW radiation at the Earth's top of atmosphere (TOA) [3-5]. 
Its successor, the Clouds and the Earth's Radiant Energy System (CERES), onboard the Terra, Aqua, Suomi National Polar-orbiting Partnership (S-NPP), and National Oceanic and Atmospheric Administration-20 (NOAA-20) satellites, not only measured the TOA radiation, but the radiative fluxes at the surface and the atmospheric radiation fluxes [6-8]. This allows for a better understanding of air-land and air-sea energy transfer and better physically based weather forecasting. CERES has provided such observations since 2000, and there will still be several CERES instruments operating in coming decades. Furthermore, the Geostationary Earth Radiation Budget (GERB) instrument onboard the Meteosat Second Generation (MSG) satellite provided the first dedicated observation of the ERB from a geostationary orbit; the generated data could help to mitigate the lack of temporal samples in the low-orbit satellite observation data of the $60^{\circ} \mathrm{S}-60^{\circ} \mathrm{N}$ region on Earth [9-11]. These satellite-based observations have enhanced our understanding of the ERB; however, they still have several limitations. A satellite-based observatory is restricted by the limited longevity of its carrying satellite. For example, the Terra satellite (one of the CERES's carrying platforms), which has the longest operational lifetime among all the current flying satellites carrying the Earth radiation observation system, will operate for 22 years and will be decommissioned around 2022 when it is due to run out of fuel [12]. The operational lifetime of the onboard instrument of around two decades could possibly be extended if the carrying satellite had longer longevity. Additionally, global radiation data from current polar-orbiting-satellite observations are time-averaging. Due to the limited temporal sampling, it may neglect some variations of small time-scales, which could be important for studies that need high temporal resolution Earth radiation data, such as research into the relationship between TOA flux and cloud fraction [13,14]. Though these polar-orbit-satellite observations can be temporally interpolated by measurements from geostationary satellites to provide better regional radiation estimates, the polar regions are not involved $[10,15]$. All of these necessitate the search for a complementary platform for the Earth's radiation observation.

The near-side of the Moon is a unique platform for observing the Earth's radiation budget [16,17]. Compared to a man-made-satellite platform, a Moon-based Earth radiation observatory (MERO) may have several advantages. As the only natural satellite of the Earth, the Moon has a much more stable orbit, which will allow for a substantially longer longevity for a Moon-based observatory than a man-made satellite. This provides the possibility for instruments deployed on the near-side of the Moon to produce longer-term and continuous data. Due to the long distance between the Moon and the Earth, a sensor on the near-side of the Moon may have an all-time near-half-globe scope. Since spatial resolution depends on the number of detectors on the focal plane of a sensor [18], if the sensor has sufficient detectors, a MERO could provide observations with abundant temporal samples and high-spatial resolution for the globe, which could help enhance the current satellite-based Earth radiation estimates. Furthermore, more accurate in situ instrument calibration can be performed due to the stable selenographic radiation environment without complications from an atmosphere. However, leaving a sensor on the Moon would face significant challenges, which mainly result from the harsh lunar environment, including the significantly large difference in the day and night temperatures ( 250 K) [16] and space radiation hazards caused by outer space high-energy particles [19]. Since man-made satellites also suffer from high-energy particles and a large day-night temperature difference in space, technologies have been developed to cope with the impacts of these two factors $[20,21]$, which could be improved and then implemented for the Moon-based instrument.

For the purpose of the prospective MERO instrumentation design, the detector's parameterization is necessary, which is closely related to the irradiance at the entrance pupil plane of the instrument. The entrance pupil irradiance (EPI) is the radiation entering the instrument, which will finally arrive at the focal plane through the optics system; in other words, the focal plane irradiance (FPI) could be derived from the EPI. The FPI is the input energy of the instrument's charge-coupled device (CCD), which converts the input radiation to a digital signal. For a specific CCD, there will be a certain device noise generated by the processing chips and circuits; thus, for the same input FPI, different CCDs will possess various signal-to-noise ratios (SNRs), and a larger SNR will lead to higher quality 
data $[18,22]$. The quantification of EPI will help with the selection of the optimal CCD with the highest SNR for a MERO instrument. Currently, however, little research has focused on EPI and the follow-on parameterization for a MERO sensor. The objective of this study is to build a model to estimate the EPIs of a MERO instrument. Section 2 is dedicated to presenting the model features, the detailed construction process, and the utilized satellite-based data. In Section 3, we present the model applications which estimated the EPIs for a hypothetic MERO located at the Apollo 15 landing site and predicted the EPIs for any given location MERO within the MERO deployable region over an 18.6-year period. Our conclusions are presented in Section 4.

\section{Model}

\subsection{General Features}

Unlike a low-orbit satellite platform, since the Moon-Earth distance $(385,000 \mathrm{~km}$ on average) is about 60 times greater than the Earth's mean radius $(6400 \mathrm{~km})$, a MERO would have a near half-globe coverage. This means that the MERO-viewed area on the Earth will have a significant curvature. Such characteristics would result in a great effect on building the MERO sensor's EPI estimating model. Due to the Moon's orbit motion and the Earth's rotation, the spatial distribution of the near-half area viewed by a MERO on the Earth is a temporal variable. Furthermore, as the Moon-Earth system orbits the Sun, it would, in general, contain a spatially varying Sun-lit part (both emitting LW and reflecting solar SW radiation to a MERO) and a non-sunlit portion (only emitting LW radiation) (see Figure 1). These will result in temporal variations of the MERO sensor's SW and LW EPIs due to the difference in the outward radiations of different Earth locations. Thus, to derive the EPIs, the temporal-spatial distribution of the MERO-viewed area at the Earth TOA and its sunlit portion should first be derived. Since the scope of a MERO covers nearly half of the globe instantaneously, the curvature of such a hemispheric surface will result in the difference in geometries of different top of atmosphere (TOA) sites towards the MERO such as the distance and the viewing zenith angle (see Figure 1), and this geometry difference will lead to a distinction in the contributions of different TOA sites in the MERO-viewed region to the MERO sensor's SW and LW EPIs.

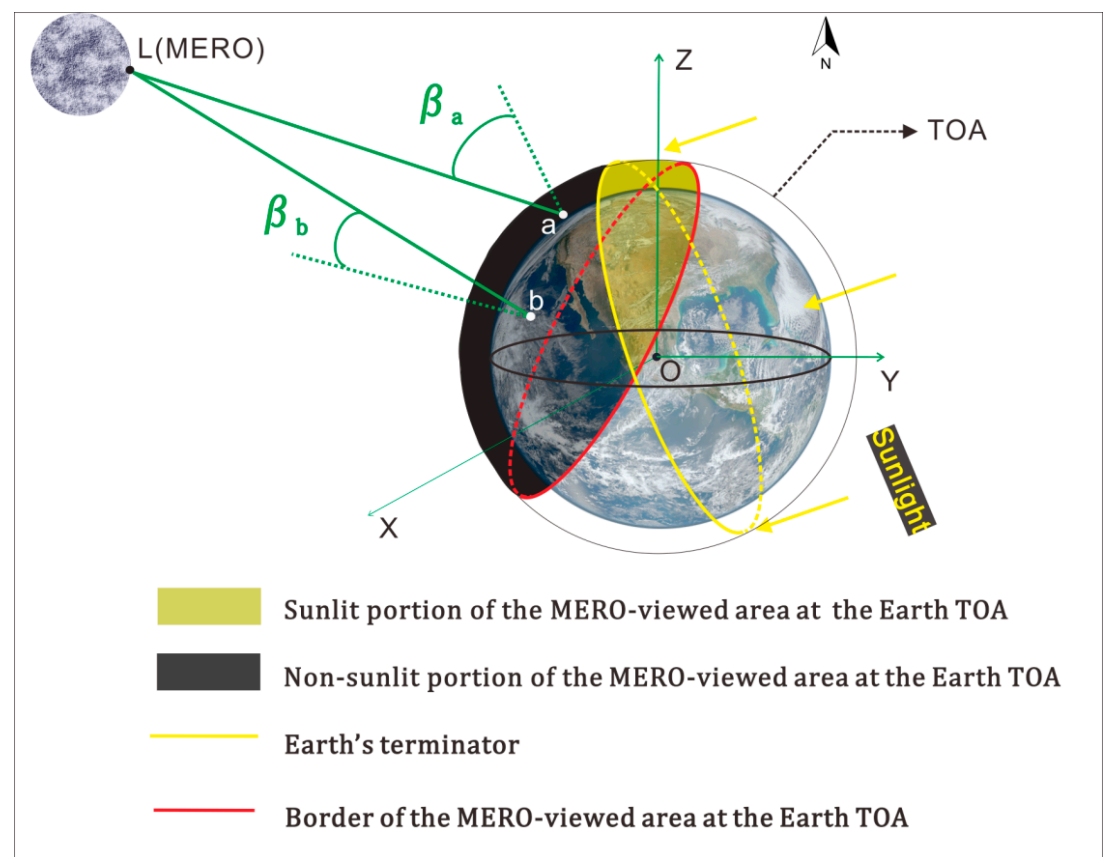

Figure 1. A sketch illustrating the sunlit and non-sunlit portions of the near-half-globe Moon-based Earth radiation observatory (MERO) viewed area at the Earth's top of atmosphere (TOA), the location-varying viewing zenith angle $\beta$ and distance to the MERO. 
As a preliminary step, only a single-pixel detector with an all-time near-half-globe scope, which has a SW broadband channel $(0.3-5 \mu \mathrm{m})$ for measuring reflected solar radiation and a LW broadband (5-200 $\mu \mathrm{m})$ channel for measuring Earth-emitted thermal radiation, is considered to be deployed in the MERO instrument. To obtain a MERO sensor's EPI, we subdivided the MERO-viewed area at the Earth TOA into finite geographic elements, and summed up the individual contributions of these elements to the MERO sensor's EPI. In this paper, such individual contributions were derived through the radiance from the subdivided TOA area to the MERO sensor with some geometric parameters by utilizing the radiation transfer function, which uses the radiance, cosine weighting and solid angle to calculate the radiation transferred from an emitter to a receiver. Therefore, the calculation of the TOA radiance toward MERO is the core part of this model, which can be derived from the TOA radiation flux and TOA anisotropic factors. Ideally, if the Earth TOA surface is Lambertian, the TOA radiance can be derived by TOA radiation flux $/ \pi$ and is isotropic, irrespective of direction. However, the actual radiance depends on the direction, which is anisotropic. Such anisotropy can be described by utilizing the TOA anisotropic factor, which is defined as actual radiance/Lambertian radiance, i.e., (actual radiance) $\times \pi /$ (TOA radiation flux). Thus, the actual TOA radiance can be derived by (TOA anisotropic factor) $\times($ TOA radiation flux) $/ \pi$, where the TOA radiation flux can be obtained from the satellite-based Earth TOA flux dataset, and the TOA anisotropic factors can be derived by referring to the CERES anisotropic factors. The CERES anisotropic factor is (actual TOA radiance) $\times \pi /$ (TOA radiation flux) and is used to convert the measured TOA radiances to the TOA radiation flux data; such usage is inverse to the utilization in our work. The CERES anisotropic factors can be generated by angular distribution models (ADMs). The CERES SW ADMs determine the SW anisotropic factors as the function of the solar zenith angle, observer zenith angle, and relative azimuth angle (defining the azimuth angle position of the observer relative to the solar plane), while the CERES LW ADMs determine the LW anisotropic factors, which depend on the observer zenith angle, since the LW anisotropy is nearly irrelative to the solar zenith angle and relative azimuth angle. Notably, the CERES ADMs have been built for a variety of scene types, and each scene type's ADMs are different. Such scene types are defined by the combination of surface type, e.g., ocean, land, with several other parameters, e.g., cloud optical depth and cloud fraction. The surface of a TOA region is defined as its directly projected area on the Earth's surface [23-25].

The steps of our construction of the MERO's EPI model are shown in Figure 2. The first procedure is to mesh the MERO-viewed area at the Earth TOA and to derive the temporal-spatial distribution of the sunlit and non-sunlit portions of such area by utilizing the NASA JPL Horizons Ephemeris System. The JPL Horizons System takes into accounts more than 100 different astronomical aspects, such as the elliptical shape of the lunar orbit, lunar librations, apsidal precession, lunar standstill cycle, and the Earth's axial precession and nutation [26]. Then, by retrieving the satellite-based Earth TOA flux dataset as a function of latitude and longitude, the TOA radiation flux of every grid node in the MERO-viewed area can be estimated. Additionally, the JPL Horizons can provide geometric parameters between the MERO and the MERO-viewed area at the Earth TOA such as distance and viewing zenith angle, which are crucial for the calculation of a MERO sensor's EPI. Finally, combined with the TOA anisotropic factors, the MERO sensor's EPIs are derived through the radiation transfer function. 


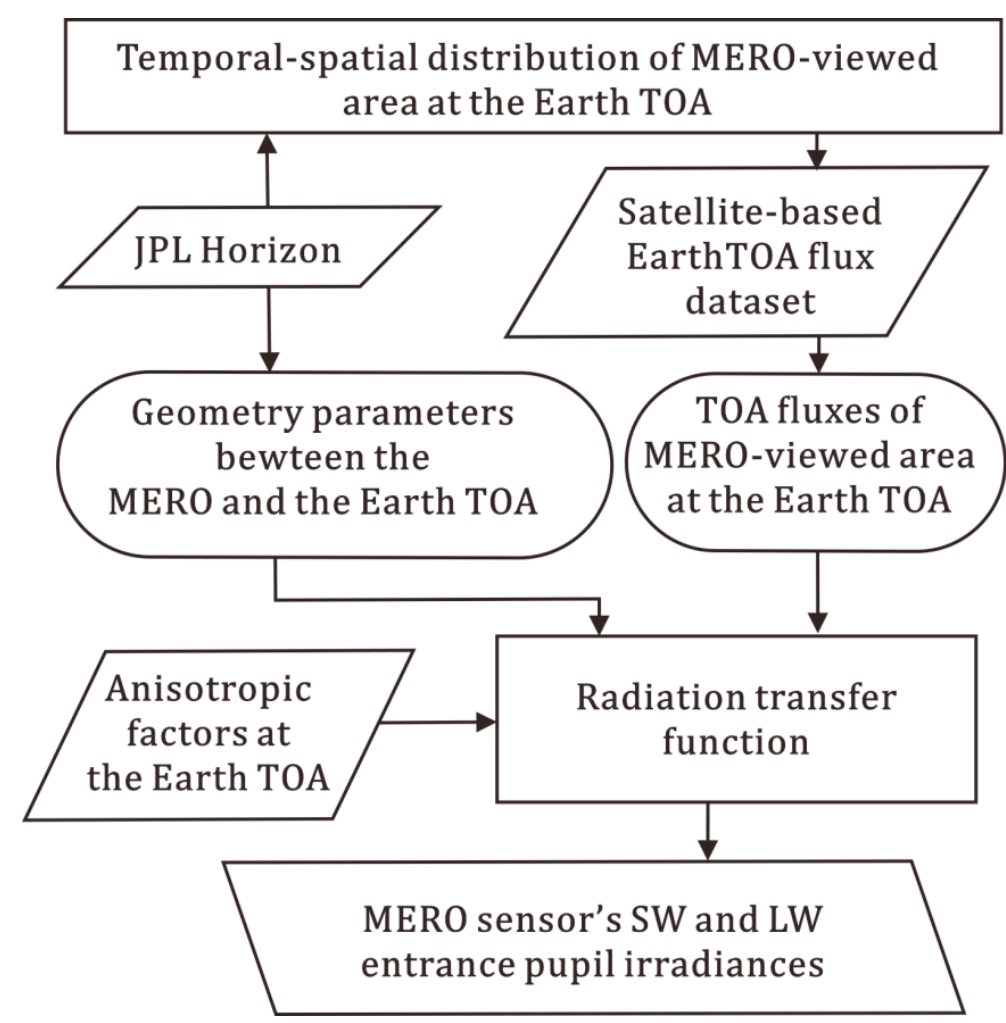

Figure 2. Flowchart of the model construction for deriving a MERO sensor's entrance pupil irradiances. SW: shortwave; LW: longwave.

\subsection{Temporal-Spatial Distribution of MERO-Viewed Area at the Earth TOA}

Figure 3 shows the process of deriving the time-dependent spatial distribution of the MERO-viewed area and its sunlit portion at the Earth TOA. The globe is discretized in $1^{\circ}$ latitude $\times 1^{\circ}$ longitude grids since the utilized satellite-based TOA flux data in this study came from CERES datasets, of which the spatial resolution is $1^{\circ}$ latitude $\times 1^{\circ}$ longitude. The MERO viewing zenith angle $\beta$ is utilized to pick out grid nodes in the MERO-viewed area on Earth by $\beta \leq 90^{\circ}$. $\beta$ can be obtained by

$$
\beta_{i}=\arccos \frac{L_{i} \cdot N_{i}}{\left|L_{i}\right|\left|N_{i}\right|}
$$

where $L_{i}$ is the MERO viewing vector of node $i$ and $N_{i}$ is the normal vector (see Figure 4). WGS-84 Cartesian coordinates of $\boldsymbol{L}_{i}$ can be derived from

$$
\begin{gathered}
\boldsymbol{L}_{i}=\left[\begin{array}{c}
D_{\mathrm{LE}} \cos \gamma \cos \varphi_{\mathrm{V}}-G_{i} \cos \theta_{i} \cos \varphi_{i} \\
D_{\mathrm{LE}} \cos \gamma \sin \varphi_{\mathrm{V}}-G_{i} \cos \theta_{i} \sin \varphi_{i} \\
D_{\mathrm{LE}} \sin \gamma-G_{i} \frac{b^{2}}{a^{2}} \sin \theta_{i}
\end{array}\right] \\
G_{i}=\frac{a}{\sqrt{1-e^{2} \sin ^{2} \theta_{i}}} \\
\gamma=\theta_{\mathrm{V}}-\arcsin \left(\frac{a e^{2} \sin \theta_{\mathrm{V}} \cos \theta_{\mathrm{V}}}{D_{\mathrm{LE}} \sqrt{1-e^{2} \sin ^{2} \theta_{\mathrm{V}}}}\right)
\end{gathered}
$$

where $a$ and $b$ are the semi-major axis and the semi-minor axis of the Earth TOA ellipsoid, which is the WGS-84 Earth ellipsoid plus the atmosphere, respectively; $e$ is the first eccentricity $\left(e=\sqrt{a^{2}-b^{2}} / a\right)$; $D_{\mathrm{LE}}$ is the distance from the Earth's center $\mathrm{O}_{\mathrm{E}}$ to the MERO location $\mathrm{L}$, which can be derived by multiplying the light speed by the parameter 399_ins_LT_LE (see Table 1) generated from the JPL 
Horizons System; $\theta_{\mathrm{V}}$ and $\varphi_{\mathrm{V}}$ are the WGS-84 latitude and longitude of the sub-MERO point V, respectively, which can be derived from the JPL Horizons System generated parameters sub-MERO latitude and sub-MERO longitude (see Table 1). Given MERO location L, the sub-MERO point V is defined as the intersection point of the Earth reference TOA ellipsoid and the Earth-MERO connection line perpendicular to this ellipsoid (see Figure 4). $\theta_{i}$ and $\varphi_{i}$ are the WGS-84 latitude and longitude of node $i$, respectively, which are given by the initial meshing. $N_{i}$ in Equation (1) can be derived from

$$
N_{i}=\left[\begin{array}{c}
\cos \theta_{i} \cos \varphi_{i} \\
\cos \theta_{i} \sin \varphi_{i} \\
\sin \theta_{i}
\end{array}\right]
$$

Thereafter, the sunlit and shadowed portions of a MERO-viewed area on Earth can be discriminated by Sun elevations $(S e)$, which can be derived from

$$
S e_{i}=90-\arccos \left(\frac{S \cdot N_{i}}{|S|\left|N_{i}\right|}\right)
$$

where $S$ is the Sun direction vector at the input time. It can be derived from

$$
S=\left[\begin{array}{c}
\cos \theta_{S} \cos \varphi_{S} \\
\cos \theta_{S} \sin \varphi_{S} \\
\sin \theta_{S}
\end{array}\right]
$$

where $\theta_{S}$ and $\varphi_{S}$ are the WGS-84 latitude and longitude of the sub-solar point $S$ at the input time. These can be achieved from the JPL Horizons System-generated parameters of sub-solar latitude and sub-solar longitude (see Table 1).

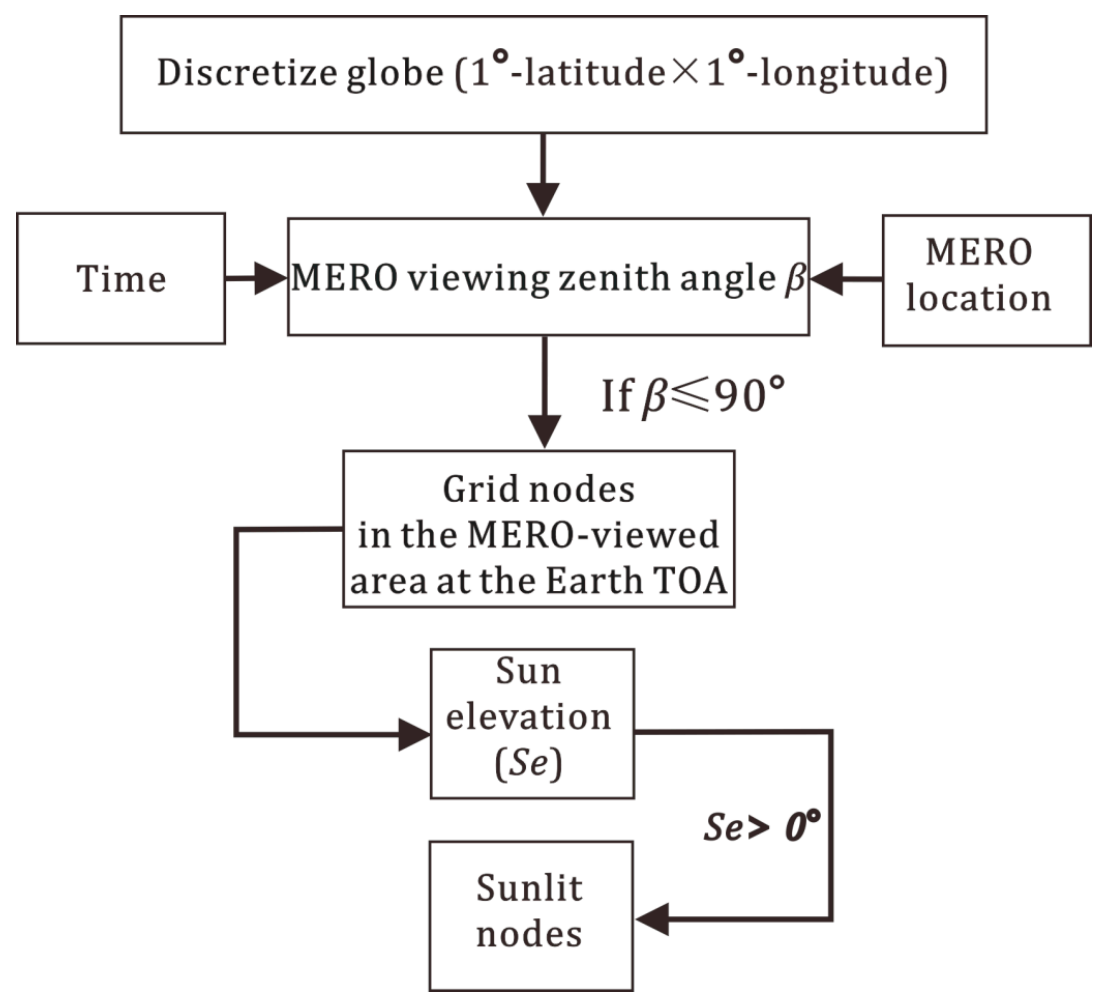

Figure 3. Flowchart to derive the temporal-spatial distribution of the MERO-viewed area at the Earth TOA. 


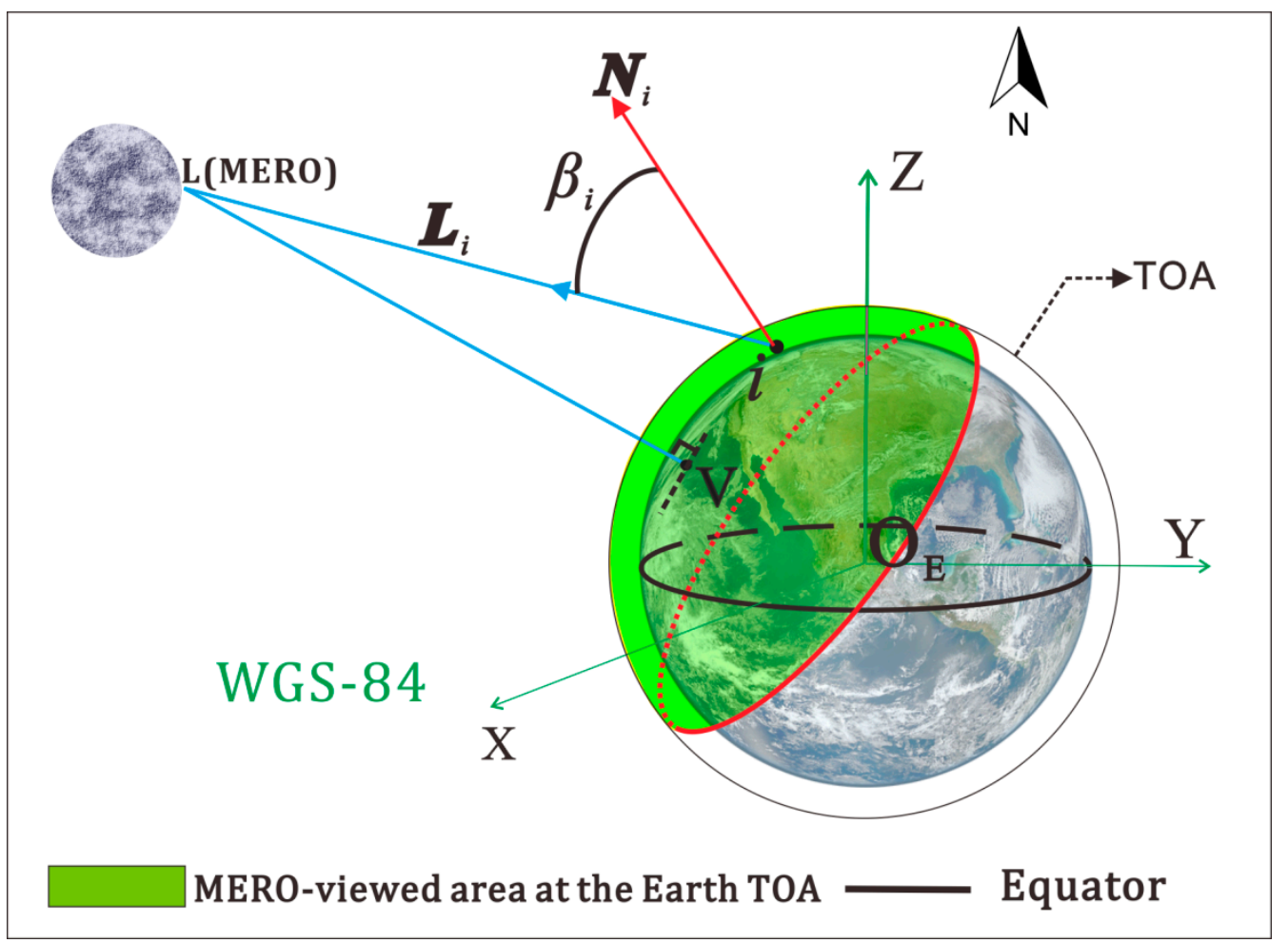

Figure 4. A sketch to illustrate the geometry of a MERO's sight line towards a grid node in the MERO-viewed area at the Earth TOA.

Table 1. The JPL Horizons System generated parameters used in this study.

\begin{tabular}{ccc}
\hline Parameter & Depiction & Unit \\
\hline Time & UTC time & \\
399_ins_LT_LE $\left(\frac{D_{L E}}{\text { light speed }}\right)$ & Instantaneous light-time of MERO location with respect to the Earth center at the set-time & Minutes \\
Sub-MERO latitude $\left(\theta_{V}\right)$ & WGS-84 latitude of the sub-MERO point V & Degree \\
Sub-MERO longitude $\left(\varphi_{\mathrm{V}}\right)$ & WGS-84 longitude of the sub-MERO point V & Degree \\
Sub-solar latitude $\left(\theta_{S}\right)$ & WGS-84 latitude of the sub-solar point $S$ & Degree \\
Sub-solar longitude $\left(\varphi_{S}\right)$ & WGS-84 longitude of the sub-solar point $S$ & Degree \\
\hline
\end{tabular}

\subsection{Radiation Transfer Function}

The rationale of this function is to sum the individual contributions of every grid node in the MERO-viewed area at the Earth TOA to the MERO instrument's EPI (see Figure 5). An individual contribution can be derived from the node's TOA radiation flux utilizing the radiation transfer function. Therefore, the MERO sensor's SW EPI $\left(\psi_{S W}\right)$ and LW EPI $\left(\psi_{L W}\right)$ can be derived through

$$
\begin{aligned}
& \psi_{S W}=\sum_{i \in S L_{N}} F s w_{i} \pi^{-1} R s w_{i} \cos \beta_{i} A_{i} \cos \eta_{i} D_{L i}^{-2} \\
& \psi_{L W}=\sum_{i \in M_{N}} F l w_{i} \pi^{-1} R l w_{i} \cos \beta_{i} A_{i} \cos \eta_{i} D_{L i}^{-2}
\end{aligned}
$$

where $F s w_{i}$ and $F l w_{i}$ are the TOA SW and TOA LW flux of node $i$, respectively, which could be derived by seeking the satellite-based TOA radiation flux data by the coordinates of node $i$. The coordinates of node $i$ have been given in the spatial distribution generation process as described in Section 2.2. In our study, the CERES SYN1deg Ed4A dataset serves as the source of the satellite-based TOA flux data [27]. CERES can provide a relatively accurate measurement of multiple Earth radiation parameters and cloud properties including the SW and LW TOA outward radiation flux. The CERES has continuously collected about 18 years of data, and in the future, there will still be a series of such instruments operating onboard. This indicates that the CERES has the potential to provide the longest-term 
continuous Earth radiation data over recent decades. The CERES data are now widely used to help us to better understand the ERB [28]. Among all of the CERES datasets, the SYN1deg-1hourly Ed4A product could provide the highest temporal resolution for the TOA flux dataset with a $1^{\circ}$ latitude $\times 1^{\circ}$ longitude spatial resolution by incorporating hourly geostationary satellite (GEO) imager data and by taking advantage of additional GEO imager channels to improve the quality of the generated TOA radiation fluxes. These hourly Earth TOA flux data could help to maximize the temporal resolution of the model-derived MERO sensor's EPI $[15,27,29]$. In Equations (8) and (9), $R s w_{i}$ and $R l w_{i}$ are the SW and LW anisotropic factors of TOA node $i$, respectively, which will be described in details in Section 2.4. $S L_{N}$ is the set of all sunlit nodes in the MERO-viewed area at the Earth TOA and $M_{N}$ is the set of all grid nodes in this area. The second term $\cos \beta_{i} A_{i}$ is the size of the projected area of node $i$ toward the MERO, where $A_{i}$ is the size of the area that node $i$ represents ( $1^{\circ}$ latitude $\times 1^{\circ}$ longitude area on Earth). The third term $\cos \eta_{i} D_{L i}^{-2}$ is the solid angle from node $i$ to per square meter of the MERO sensor's entrance pupil surface. $D_{L i}$ is the distance between the MERO and node $i$, which is the length of $L_{i}$ and can be derived from Equations (2)-(4). $\eta_{i}$ is the angle between $L_{i}$ and the MERO instrument orientation vector $L_{V}$ (see Figure 5), where $L_{V}$ can be used to represent the instrument's orientation since a MERO sensor always faces straight toward the sub-MERO point V. $\eta_{i}$ can be derived from

$$
\eta_{i}=\arccos \frac{\boldsymbol{L}_{i} \cdot \boldsymbol{L}_{\mathrm{v}}}{\left|\boldsymbol{L}_{i}\right|\left|\boldsymbol{L}_{\mathrm{V}}\right|}
$$

where $L_{i}$ can be derived from Equations (2)-(4), and $L_{\mathrm{V}}$ can be derived from

$$
\begin{gathered}
\boldsymbol{L}_{\mathrm{V}}=\left[\begin{array}{c}
D_{\mathrm{LE}} \cos \gamma \cos \varphi_{V}-G_{\mathrm{V}} \cos \theta_{\mathrm{V}} \cos \varphi_{\mathrm{v}} \\
D_{\mathrm{LE}} \cos \gamma \sin \varphi_{V}-G_{\mathrm{V}} \cos \theta_{\mathrm{v}} \sin \varphi_{\mathrm{v}} \\
D_{\mathrm{LE}} \sin \gamma-G_{\mathrm{v}} \frac{b^{2}}{a^{2}} \sin \theta_{\mathrm{v}}
\end{array}\right] \\
G_{\mathrm{V}}=\frac{a}{\sqrt{1-e^{2} \sin ^{2} \theta_{\mathrm{v}}}}
\end{gathered}
$$

where $\gamma$ can be derived from Equation (4). $D_{\mathrm{LE}}, \theta_{\mathrm{v}}$, and $\varphi_{\mathrm{v}}$ are given by the parameters 399_ins_LT_LE, sub-MERO latitude, and sub-MERO longitude, respectively, from the JPL Horizons System (see Table 1). 


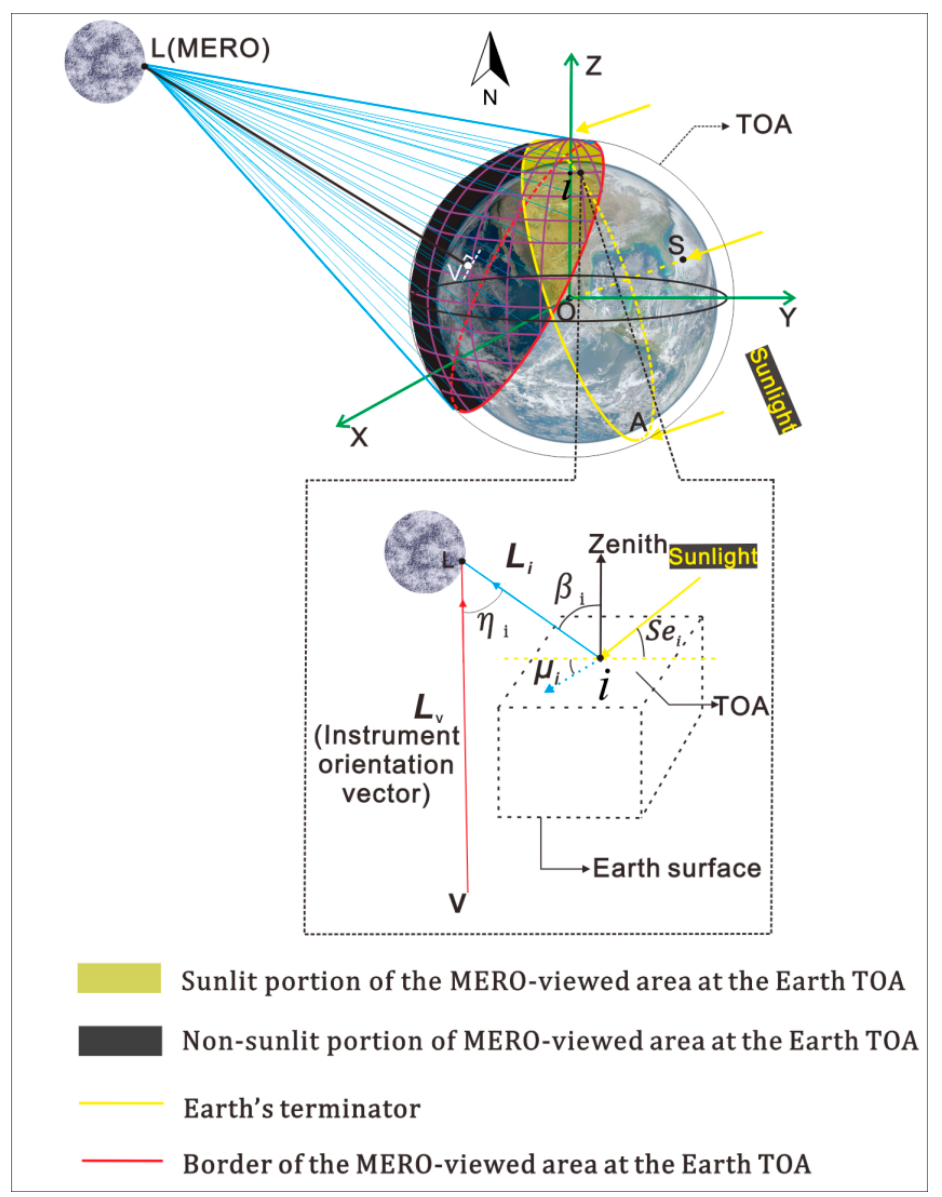

Figure 5. A sketch to illustrate the function for calculating the MERO sensor's entrance pupil irradiances. $\beta_{i}$ is the MERO viewing zenith angle; $S e_{i}$ is the Sun elevation; $\mu_{i}$ is the relative azimuth angle; $\eta_{i}$ is the MERO zenith angle of grid node $i$ in the MERO-viewed area at the Earth TOA; and V is the sub-MERO point.

\subsection{Earth TOA Anisotropic Factors}

In our study, the TOA SW anisotropic factor (Rsw) and TOA LW anisotropic factor (Rlw) are derived with several simplifications based on the CERES ADMs:

1. Since it is not feasible for us to establish a reasonable function to characterize the SW radiance anisotropy based on the CERES ADM references [23,25], at this stage of research, the SW radiance exiting the Earth TOA is assumed to be isotropic, thus Rsw is 1 constantly;

2. According to the CERES LW ADMs [25], the TOA LW anisotropic factor Rlw is determined by a set of viewing-zenith-angle-dependent functions under several scene types. We simplified these scene types as a combination of surface types (ocean, land, desert, and snow) with two cloud conditions (clear sky and cloudy sky), and the surface of a TOA node is defined as the $1^{\circ}$ latitude $\times 1^{\circ}$ longitude area at the Earth's surface directly beneath this node. The clear condition is defined by cloud coverage $f \leq 0.1 \%$. $f$ could be derived from the CERES SYN1deg Ed4A dataset.

For the TOA grid nodes of the ocean, land, and desert surface types, the Rlw, as the function of the MERO viewing zenith angle $(\beta)$, is shown in Figure $6 \mathrm{a}$. The $R l w$ ranges from $\sim 0.78$ to $\sim 1.04$ under the clear condition, and from $\sim 0$ to $\sim 1.18$ under the cloudy condition. A value of 1.04 implies that the actual radiance is bigger than the ideally Lambertian radiance at the nadir direction, as does the value of 1.18, while for the TOA nodes with the snow surface type, the Rlw against $\beta$ under the clear condition is very similar to that under the cloudy condition (see Figure 6b). 

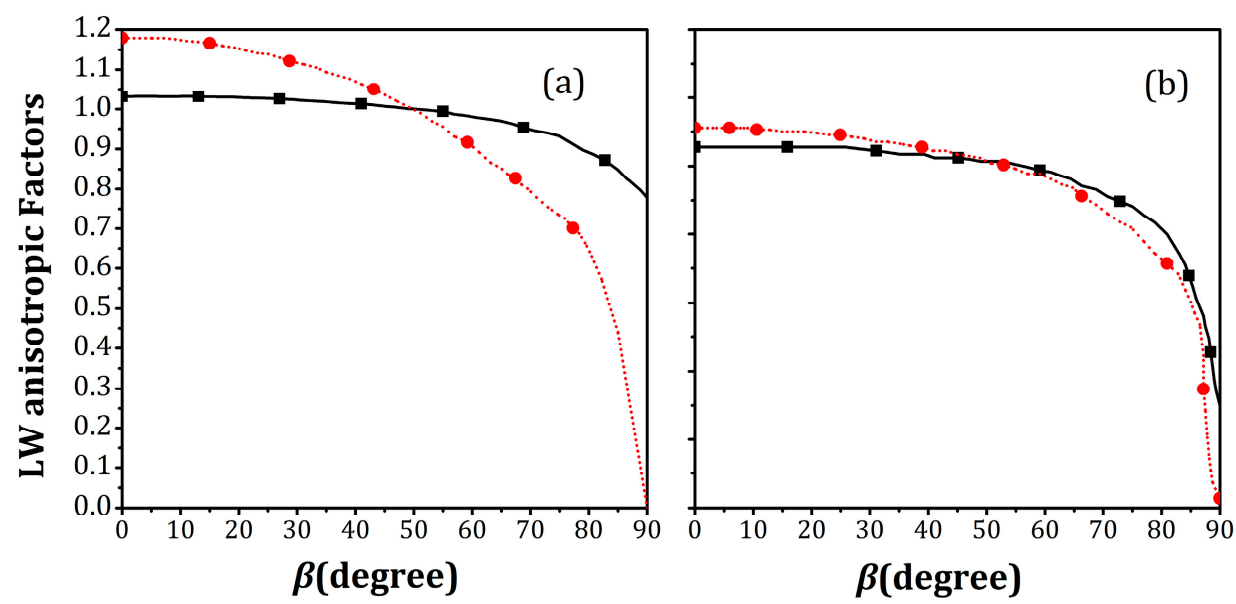

$\longrightarrow$ Clear sky $\cdots \cdot \cdots \cdot \ldots$ Cloudy sky

Figure 6. Earth TOA LW anisotropic factors for (a) the land, ocean, and desert surface types; and (b) the snow surface type, as a function of the MERO viewing zenith angle $\beta$.

The surface type of every TOA grid node in the MERO-viewed area is determined using the CERES surface type data [30], which utilize 18 categories with IDs from 1 to 18 to represent the Earth's surface with a spatial resolution of $1^{\circ}$ latitude $\times 1^{\circ}$ longitude. In our study, except for the TOA grid nodes with an ID of 15, which represented the snow surface type, others are defined as the TOA nodes with land, ocean, and desert surface types.

\section{Application Results and Discussion}

\subsection{EPIs over the Moon's Orbital Period}

As a model application, the SW and LW EPIs of a hypothetic MERO instrument located at the Apollo 15 landing site (selenographic coordinates: $3.628^{\circ} \mathrm{E}, 26.133^{\circ} \mathrm{N}$ ) over the Moon's orbital period are evaluated. Results show that the SW EPI ranged from about 0 to $0.065 \mathrm{~W} / \mathrm{m}^{2}$, while for the LW EPI, the maximum value was about $0.075 \mathrm{~W} / \mathrm{m}^{2}$ and the minimum was about $0.061 \mathrm{~W} / \mathrm{m}^{2}$ (see Figure 7a,b). The latitude of the sub-MERO point on Earth of this hypothetical MERO over the Moon's orbital period from October 1 to October 29, 2017 is shown in Figure 7c. The positive latitude means that the sub-MERO point V is on the Earth's Northern Hemisphere, whereas the negative latitude means the sub-MERO point V is on the Earth's Southern Hemisphere. Since the sub-MERO point is the center of the MERO-viewed area, the Northern-Hemisphere-located-V would imply that the MERO-viewed area at the Earth TOA is primarily situated in the Northern Hemisphere, while the Southern-Hemisphere-located-V indicates that the MERO-viewed area at the Earth TOA is primarily situated in the Southern Hemisphere. A comparison of Figure 7a-c indicates that the SW EPI during the period when the sub-MERO point $V$ is located in the Northern Hemisphere is greater than that during the Southern-Hemisphere-located-V period. This is probably because the SW reflectivity of the land is greater than that of the ocean, and the Northern Hemisphere has a larger land fraction than the Southern Hemisphere. This finding is also applicable to the LW EPI because the TOA LW flux distributions in the Northern Hemisphere and Southern Hemisphere were not symmetrical over October 2017 (see Figure 8); i.e., although the TOA LW flux in the $0-60^{\circ} \mathrm{N}$ region was symmetrical to that in the area of $0-60^{\circ} \mathrm{S}$, the Arctic region had a bigger value than the Antarctic region. If the sub-MERO point $(\mathrm{V})$ was in the Northern Hemisphere (more of the Arctic zone was included in the MERO-viewed area than the Antarctic zone), more LW radiation will be emitted to the MERO sensor than the scene where V was located in the Southern Hemisphere (more of the Antarctic zone was included in the MERO-viewed area than the Arctic zone), since the MERO-viewed area covers almost half of the globe. 

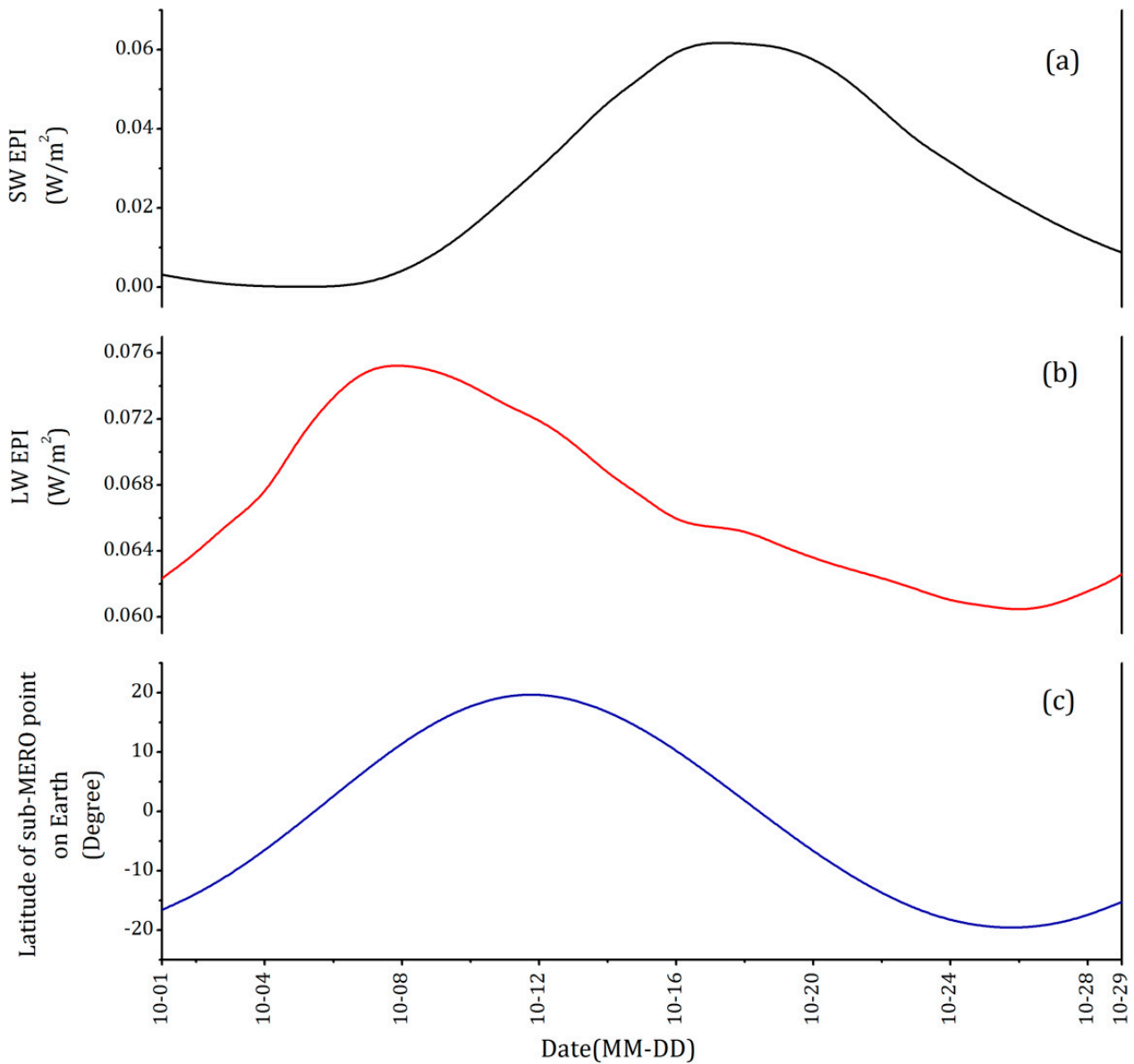

Figure 7. SW (a), LW (b), entrance pupil irradiance (EPI) and the latitude of the sub-MERO point on Earth (c), of the hypothetic MERO located at the Apollo 15 landing site over about the Moon's orbital period from October 1 to October 29, 2017.

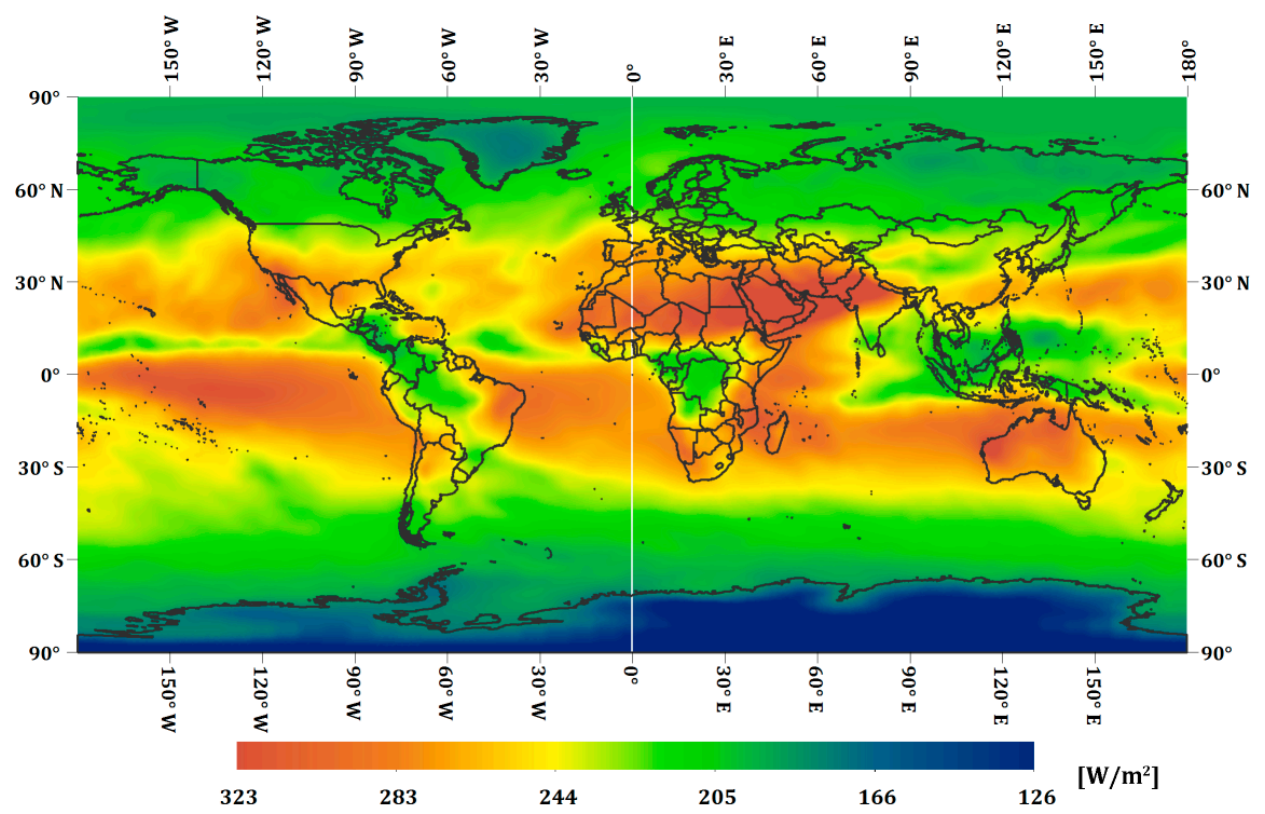

Figure 8. Monthly average Earth TOA LW flux on October 2017 derived from the CERES SYN1deg Ed4A dataset. 


\subsection{EPIs over the Future 18.6 Years}

In order to predict SW and LW EPIs for any MERO sensor at any given location over the future 18.6 years (a major lunar standstill cycle), the previously derived EPI of the hypothetic MERO instrument located at the Apollo 15 landing site during the month of October 2017 is set as the benchmark; then, anomalies relative to this benchmark, resulting from the Earth TOA flux variation, the shift of the Moon-Earth orbit, and various MERO selenographic locations over the next 18.6 years is estimated. These three factors are evaluated for prediction since they are likely to be the predominant factors involved in the variation of a MERO sensor's EPIs. The month of October 2017 is chosen as the benchmark month since data from this period are the most recent among the CERES SYN1deg-1hourly Ed4A archive, which is the TOA flux data source for the model. Hereafter, the MERO located at the Apollo 15 landing site is abbreviated as A15 MERO.

\subsubsection{Earth TOA Flux}

Variation of the MERO sensor's EPI is sensitive to the Earth's TOA flux change. Ascertaining such sensitivity would facilitate predicting anomalies in the MERO sensor's EPI relative to the benchmark, resulting from the Earth's TOA flux perturbation in the future. We quantify this sensitivity by the MERO sensor's EPI response as a function of the uniform increase of global Earth TOA fluxes. The MERO sensor's EPI response (\%) is defined as the monthly mean of the A15 MERO sensor's EPI perturbation divided by this sensor's EPI over October 2017. Therefore, for the uniform increase of different global TOA fluxes, e.g., $2 \mathrm{~W} / \mathrm{m}^{2}$ and $4 \mathrm{~W} / \mathrm{m}^{2}$, denominators of both of their EPI responses are the same, whereas the numerator of the EPI response of $4 \mathrm{~W} / \mathrm{m}^{2}$ TOA flux increase is twice as large as that of $2 \mathrm{~W} / \mathrm{m}^{2}$, making the EPI response in relative terms (\%) to the uniform increase of global Earth TOA fluxes in absolute terms $\left(\mathrm{W} / \mathrm{m}^{2}\right)$ linear for both the SW and LW. This sensitivity for SW was found to be a $1 \mathrm{~W} / \mathrm{m}^{2}$ global TOA flux uniform increase, resulting in about a $0.85 \%$ augmentation in the MERO sensor's EPI, whereas a $1 \mathrm{~W} / \mathrm{m}^{2}$ global TOA LW flux uniform increase would lead to approximately a $0.38 \%$ augmentation in the LW EPI. SW sensitivity is about twice as large as LW sensitivity, most likely because the denominator of the EPI response is the sensor's EPI, which is derived from the TOA flux (Equations (8) and (9)), as indicated by the CERES Energy Balanced and Filled (EBAF) dataset, and the global mean TOA SW flux was $\sim 100 \mathrm{~W} / \mathrm{m}^{2}$, whereas the LW was $\sim 200 \mathrm{~W} / \mathrm{m}^{2}$ (see Figure 9), which would make the SW EPI response about twice as large as the LW EPI response for the same amount of uniform increase of global TOA fluxes.

As suggested by the CERES global mean TOA flux data from the CERES EBAF dataset [31], over the past 17 years from March 2000 to October 2017, both the TOA SW and LW flux variations were periodic oscillations with a nearly stable peak-to-peak amplitude and were within -9 to $10 \mathrm{~W} / \mathrm{m}^{2}$ and -5 to $5 \mathrm{~W} / \mathrm{m}^{2}$, respectively, relative to the value of the benchmark month of October 2017 (see Figure 9). This stable-peak-to-peak-amplitude periodic oscillation indicates that although there are many factors such as atmospheric aerosol changing and snow cover variation which have consequences for the TOA flux, the sum of these effects may be small. This suggests a possibility of the same periodical variation of TOA flux over the next 18.6-year lunar standstill cycle as the past 17 years. Therefore, when considering the sensitivity depicted above, it can be concluded that the corresponding MERO sensor's SW and LW EPI anomalies would be within -7.65 to $8.50 \%$ and -1.90 to $1.90 \%$ relative to the benchmark, respectively, over the period from October 2017 to June 2036. 

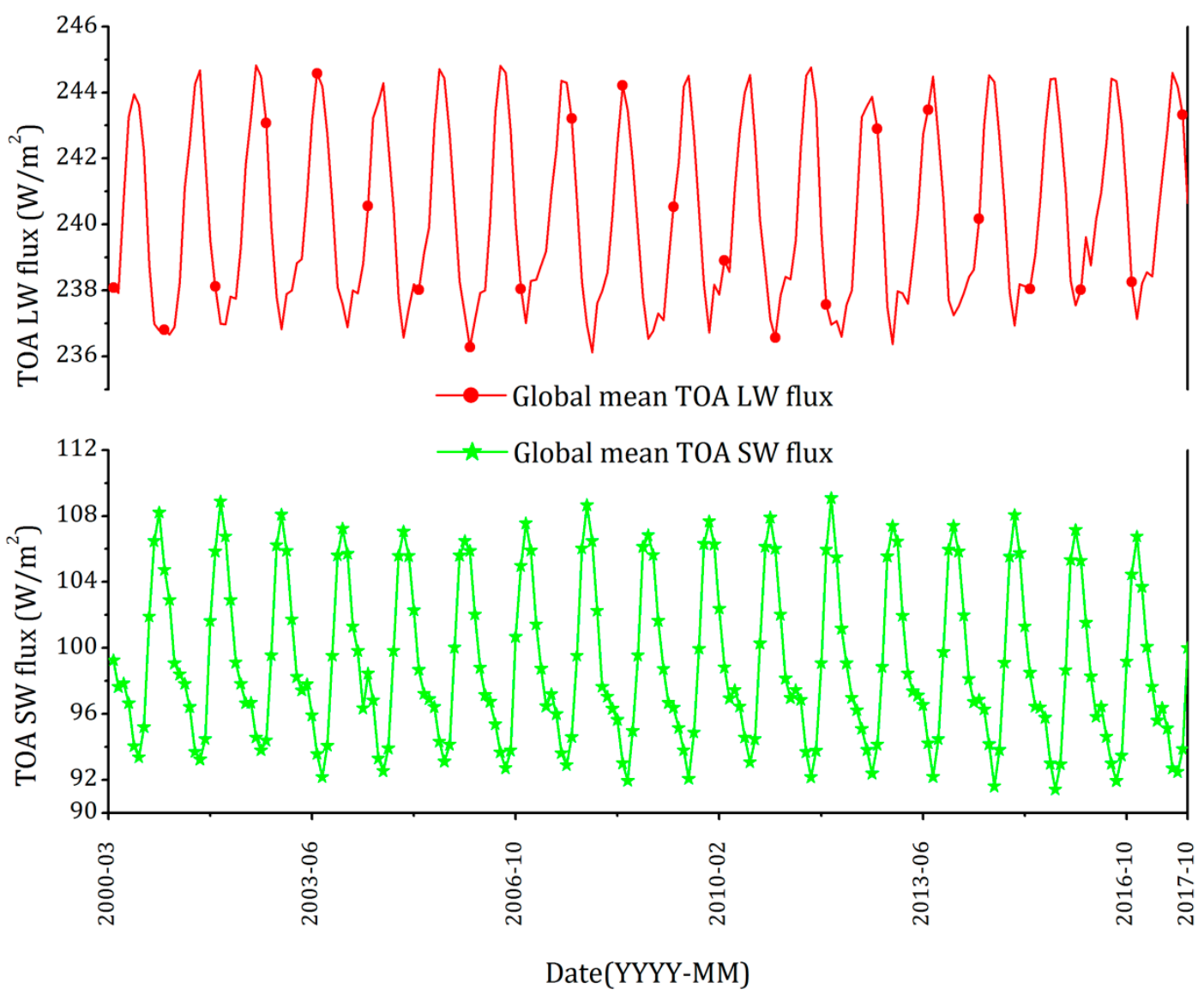

Figure 9. Global mean Earth TOA SW and LW fluxes from March 2000 to October 2017 derived from the CERES EBAF dataset.

\subsubsection{The Moon-Earth Orbit}

Due to the variation of the angle between the orbit of the Moon and the Earth's Equator $\left(18.30^{\circ}\right.$ to $28.60^{\circ}, 18.6$-year cycle), a MERO-viewed area on Earth appears to shake up and down during an 18.6-year lunar standstill cycle, which will result in differences between the MERO sensor's EPI at different Moon orbital periods. Furthermore, the apsidal precession (8.7-year cycle) of the Moon's orbit would also lead to such differences. In order to characterize the anomaly in the MERO's EPI relative to the benchmark resulting from the shift of the Moon-Earth orbit, the distribution of the Earth TOA flux is assumed as temporally constant, since the Earth TOA flux temporal variation can also cause a change to the MERO sensor' EPI. Specifically, during the next lunar standstill cycle (18.6 years), this temporally constant Earth TOA flux is set as the monthly mean of the benchmark month October 2017 from the CERES SYN1 degree dataset. This monthly mean flux is chosen as it can make the TOA SW flux available all over the globe during the future 18.6 years. The all-time globally available SW TOA flux is crucially required due to the fact that the spatial distribution of the sunlit portion of the MERO-viewed area at the Earth TOA is temporally varying and this sunlit portion may sometimes not have location-matched TOA SW fluxes without the all-time globally available TOA SW flux data. Figure 10 illustrates the model-derived monthly maximum and minimum EPIs of the A15 MERO sensor over a lunar standstill period from October 2017 to June 2036. During the benchmark month of October 2017, the monthly maximum MERO sensor's SW EPI is about $0.055 \mathrm{~W} / \mathrm{m}^{2}$; however, due to the Moon's orbital variation, it would increase up to about $0.075 \mathrm{~W} / \mathrm{m}^{2}$ over the following 18.6 years (Figure 10a), whereas the minimum of this $\mathrm{SW}$ irradiance remains at approximately $0 \mathrm{~W} / \mathrm{m}^{2}$. For the MERO sensor's LW EPI, the maximum value over October 2017 is $0.077 \mathrm{~W} / \mathrm{m}^{2}$, and will increase up to about $0.082 \mathrm{~W} / \mathrm{m}^{2}$ over the next 18.6 years; the minimum value of the benchmark month is 
approximately $0.062 \mathrm{~W} / \mathrm{m}^{2}$ and that of the future 18.6 years will decrease to $0.058 \mathrm{~W} / \mathrm{m}^{2}$ (Figure $10 \mathrm{~b}$ ). These indicate that, over the period from October 2017 to June 2036, the Moon's orbital variation would result in up to about a 36.36\% increase in the maximum A15 MERO sensor's SW EPI, and up to about a $6.49 \%$ increase and 6.45\% decrease in the maximum and minimum A15 MERO sensor's LW EPIs, respectively, relative to the benchmark, which is the A15 MERO sensor's EPI over October 2017.

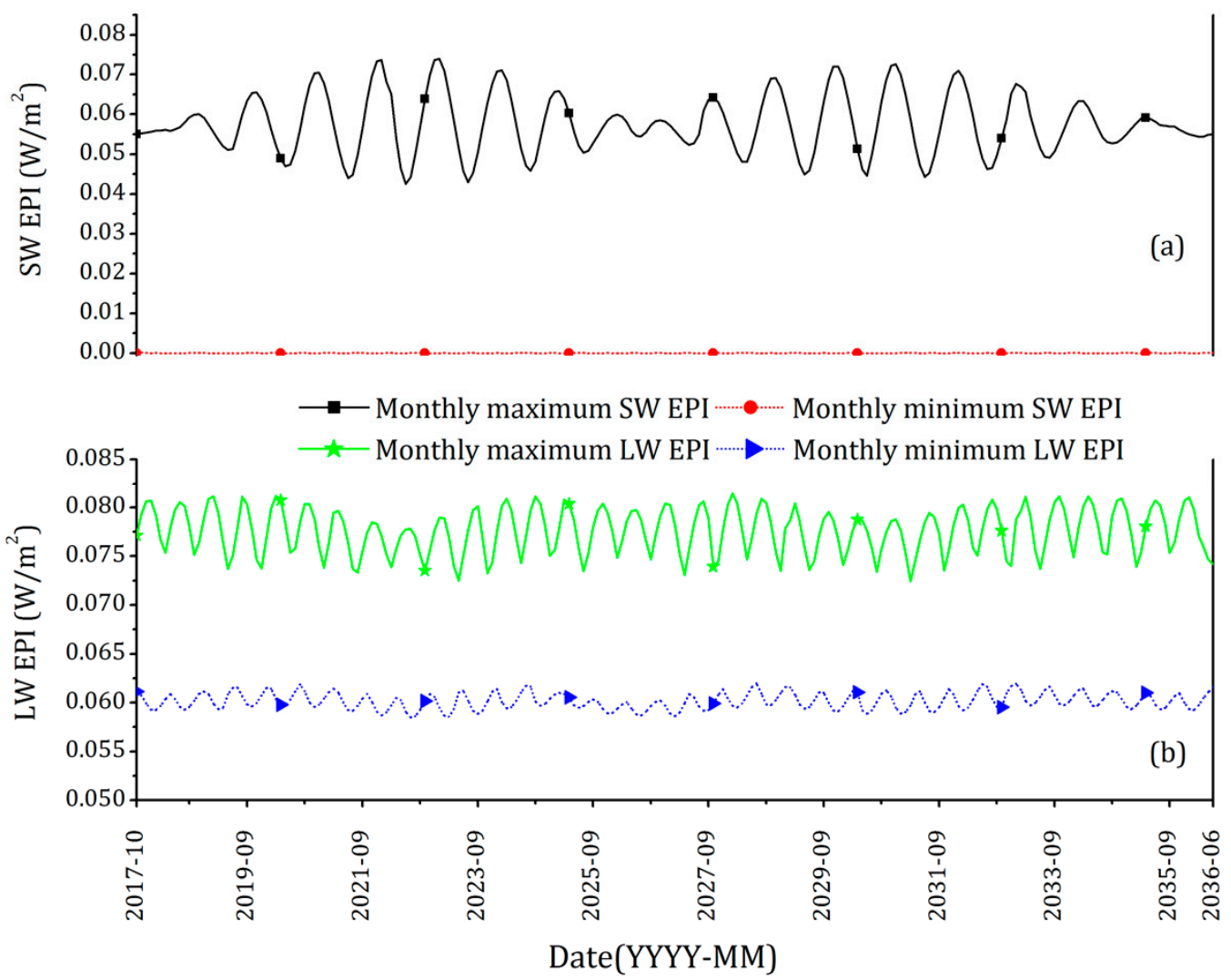

Figure 10. (a) Monthly maximum and minimum SW entrance pupil irradiances (EPIs); (b) monthly maximum and minimum LW EPIs of a hypothetical MERO sensor located at the Apollo 15 landing site over a major lunar standstill cycle from October 2017 to June 2036 with an assumption of temporally constant Earth TOA SW and LW fluxes.

\subsubsection{Selenographic Location}

Different MERO locations on the near-side of the Moon would result in a difference in the instantaneous MERO-viewed area at the Earth TOA, leading to a difference in the MERO sensor's EPI. Since the MERO's deployable region is within the area of $80.5^{\circ} \mathrm{W}-80.5^{\circ} \mathrm{E}$ and $81.5^{\circ} \mathrm{S}-81.5^{\circ} \mathrm{N}$ on the near-side of the Moon [32], to illustrate the extreme scene, two location pairs-the EW pair $\left(\mathrm{ME}, 80^{\circ} \mathrm{E}\right.$, $0^{\circ}$ and $\left.\mathrm{MW}, 80^{\circ} \mathrm{W}, 0^{\circ}\right)$ and $\mathrm{NS}$ pair $\left(\mathrm{MN}, 0^{\circ}, 81^{\circ} \mathrm{N}\right.$ and $\left.\mathrm{MS}, 0^{\circ}, 81^{\circ} \mathrm{S}\right)$-are utilized. The EW pair is used to evaluate the difference in the MERO sensor's EPI resulting from the maximum longitudinal selenographic distance between two MEROs, and the NS pair is for the evaluation of the maximum latitudinal distance. Due to the temporal variation of the MERO-viewed area at the Earth TOA, such differences will change with time. The MERO sensor's EPI differences resulting from the EW pair and NS pair over the Moon's orbital period (October 1, 2017 to October 29, 2017) are illustrated in Figure 11, which shows that the NS pair will cause up to a 36.10\% difference in the MERO sensor's SW EPI, that was higher than the maximum value $(20.82 \%)$ resulting from the EW pair. However, for the MERO sensor's LW EPI, this difference is much smaller, with the maximum value resulting from the EW pair $(0.23 \%)$ being slightly higher than that from the NS pair $(0.20 \%)$. 


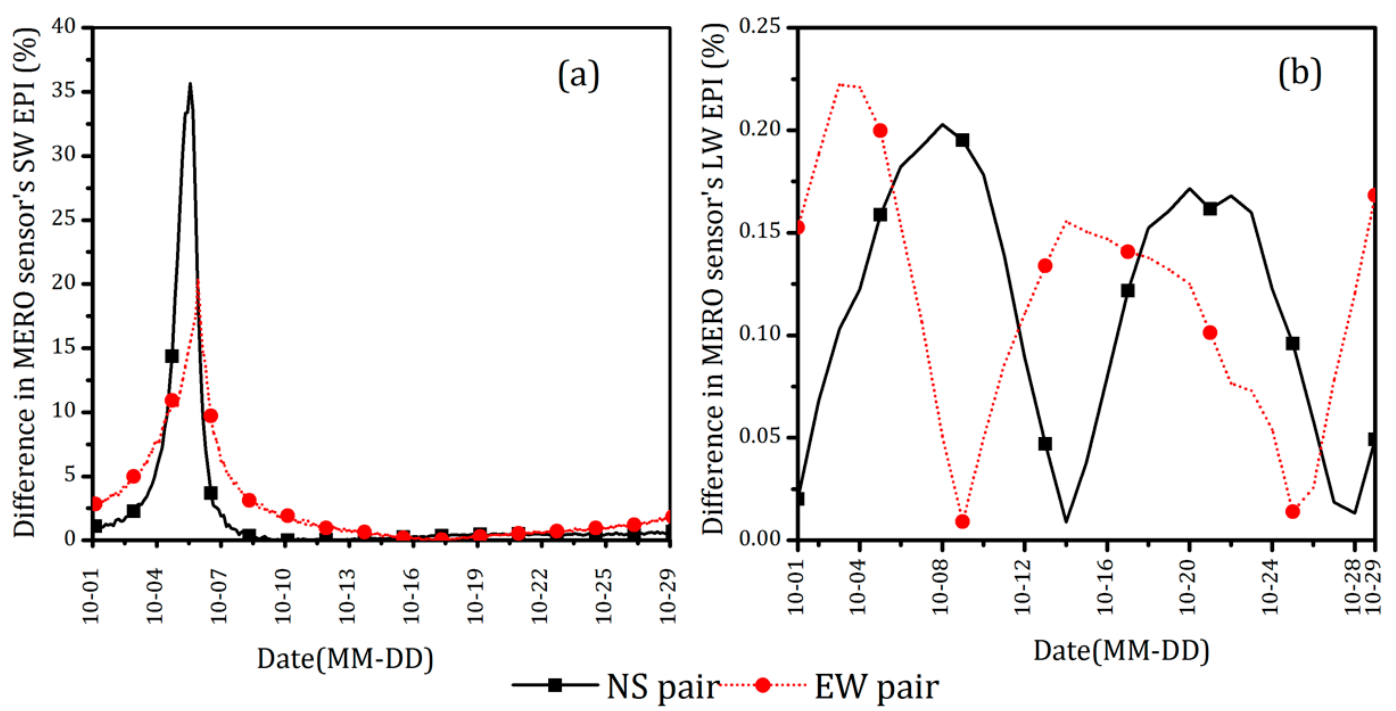

Figure 11. Time series of (a) differences in the MERO sensor's SW entrance pupil irradiance (EPI) and (b) differences in the LW EPI, resulting from the hypothetical EW MERO location pair and from the NS MERO location pair over a Moon's orbital period from October 1, 2017 to October 29, 2017.

As indicated above, over the following 18.6 years from October 2017 to June 2036, relative to the benchmark (which is the A15 MERO sensor's EPI over October 2017), the anomaly in the MERO sensor's SW EPI resulting from the Earth TOA flux variations is within -7.65 to $8.50 \%$ and that in the MERO sensor's LW EPI is within -1.9 to $1.9 \%$. The Moon's orbital variation would lead to up to about a $36.36 \%$ increase in the maximum MERO sensor's SW EPI, and up to an approximate $6.49 \%$ increase and $6.45 \%$ decrease in the maximum and minimum MERO sensor's LW EPIs, respectively, relative to the benchmark. Furthermore, the anomaly in the MERO sensor's SW EPI resulting from the MERO's deployment location is up to $36.10 \%$ relative to the benchmark, and that in the MERO sensor's LW EPI is up to $0.23 \%$. All of these suggest that the maximum sensor's SW EPI of a MERO, which can be located anywhere within the MERO deployable region, over the future 18.6 years will be greater than the maximum of the benchmark by less than $80.96 \%$. Note that the maximum and minimum SW EPIs of the benchmark are 0.065 and $0 \mathrm{~W} / \mathrm{m}^{2}$, respectively (Figure 7). On the other hand, for the sensor's LW EPI, such an increase in the maximum value and decrease in the minimum value compared to the benchmark would be less than $8.62 \%$ and $8.58 \%$, respectively. In particular, the maximum and minimum LW EPIs of the benchmark are about 0.075 and $0.061 \mathrm{~W} / \mathrm{m}^{2}$, respectively. In summary, over the next lunar standstill period from October 2017 to June 2036, the sensor's SW EPI of any given location MERO, located within the MERO deployable region, would vary between 0 and $0.118 \mathrm{~W} / \mathrm{m}^{2}$, while the range of the MERO sensor's LW EPI is from 0.056 to $0.081 \mathrm{~W} / \mathrm{m}^{2}$.

In this paper, we established a framework for the MERO sensor's EPI estimation. However, a limitation still exists in the model. The SW radiance exiting the Earth TOA is currently set as isotropic when in fact it should be anisotropic. In order to estimate the EPI more precisely, a function to reasonably characterize such anisotropy is crucially needed.

\section{Conclusions}

A Moon-based Earth radiation observatory (MERO) could provide longer-term continuous measurements of radiation exiting the Earth system when compared to the current satellite-based platforms. For the parameterization of a MERO sensor, quantifying the entrance pupil irradiances (EPI) is of great importance. However, little research has focused on such EPI. In this study, we built a shortwave (SW) $(0.3-5 \mu \mathrm{m})$ and longwave (LW) $(5-200 \mu \mathrm{m})$ EPI estimating model for a simplified single-pixel MERO sensor. For the model application, the sensor's EPIs for a hypothetic MERO located at the Apollo 15 landing site were evaluated. The results showed that the SW EPI would vary from 
0 to $0.065 \mathrm{~W} / \mathrm{m}^{2}$, and the LW EPI ranged between 0.061 and $0.075 \mathrm{~W} / \mathrm{m}^{2}$ from October 1 to October 29 , 2017. Additionally, we also utilized this model to predict the SW and LW EPIs for a MERO at any given location within the MERO deployable region (region of $80.5^{\circ} \mathrm{W}-80.5^{\circ} \mathrm{E}$ and $81.5^{\circ} \mathrm{S}-81.5^{\circ} \mathrm{N}$ on the near-side of the Moon) over the future 18.6 years. The results suggest that the SW EPI will vary between 0 and $0.118 \mathrm{~W} / \mathrm{m}^{2}$, while the range of the LW EPI is from 0.056 to $0.081 \mathrm{~W} / \mathrm{m}^{2}$. Note that the magnitudes of a MERO sensor's SW and LW EPIs are not significantly different from the CERES instrument flying onboard the Terra, Aqua and Suomi-NPP satellites, of which both the magnitudes of the SW and LW EPIs are of the magnitude of the order of about $10^{-2} \mathrm{~W} / \mathrm{m}^{2}$ [33]. This suggests that current technology could meet the requirement of the MERO sensor's design specification in EPI.

In the actual design, a MERO sensor should have multiple pixels either for the SW and LW channel, since the spatial resolution depends on the number of the pixels. There is spatial-temporal variability in the Earth radiation. The multi-pixel non-scanner MERO instrument has the potential to provide long-term continuous data with adequate spatial resolution to characterize such spatial dependence. Though this study only established an EPI estimating model for a simplified single-pixel sensor, this model could still be implemented to ascertain the pixel-scale EPI for the prospective multiple-pixel non-scanner instrument with some improvements. The most important next step is to ascertain the temporal spatial distribution of the relatively smaller pixel-viewed area on Earth, which can be derived from the combination of the MERO-viewed area positioning approach that we proposed in this study and the pixel array structure.

Author Contributions: Conceptualization, W.D. and S.H.; methodology, W.D.; software, W.D.; validation, W.D., S.H., and C.N.; formal Analysis, W.D.; investigation, W.D. and C.N.; writing-original draft preparation, W.D.; writing-review and editing, W.D., S.H., and C.N.; project administration, S.H.; funding acquisition, W.D., S.H., and C.N.

Funding: This research was funded by the National Natural Science Foundation of China (grant number 41590855) and by the China Scholarship Council (grant number 201706280348).

Acknowledgments: We thank Xianglei Huang and Yisuan Chen from the Department of Climate and Space Science and Engineering of University of Michigan for their assistance. The Jet Propulsion Lab (JPL) Horizons data used in this study were downloaded from the online ephemeris system at https://ssd.jpl.nasa.gov/?horizons. The CERES SYN1deg Ed4A dataset was downloaded from the online CERES data ordering system at https: //ceres.larc.nasa.gov/order_data.php.

Conflicts of Interest: The authors declare no conflict of interest.

\section{References}

1. Wielicki, B.A.; Wong, T.; Allan, R.P.; Slingo, A.; Kiehl, J.T.; Soden, B.J.; Gordon, C.T.; Miller, A.J.; Yang, S.K.; Randall, D.A.; et al. Evidence for large decadal variability in the tropical mean radiative energy budget. Science 2002, 295, 841-844. [CrossRef] [PubMed]

2. Pinker, R.T.; Zhang, B.; Dutton, E.G. Do satellites detect trends in surface solar radiation? Science 2005, 308, 850-854. [CrossRef] [PubMed]

3. Luther, M.R.; Cooper, J.E.; Taylor, G.R. The Earth Radiation Budget Experiment Nonscanner Instrument. Rev. Geophys. 1986, 24, 391-399. [CrossRef]

4. Barkstrom, B.R.; Smith, G.L. The Earth Radiation Budget Experiment: Science and implementation. Rev. Geophys. 1986, 24, 379-390. [CrossRef]

5. Kopia, L.P. Earth Radiation Budget Experiment Scanner Instrument. Rev. Geophys. 1986, 24, 400-406. [CrossRef]

6. Wielicki, B.A.; Barkstrom, B.R.; Baum, B.A.; Charlock, T.P.; Green, R.N.; Kratz, D.P.; Lee, R.B.; Minnis, P.; Smith, G.L.; Wong, T. Clouds and the Earth's Radiant Energy System (CERES): Algorithm overview. IEEE Trans. Geosci. Remote Sens. 1998, 36, 1127-1141. [CrossRef]

7. Wielicki, B.A.; Barkstrom, B.R.; Harrison, E.F.; Lee, R.B., III; Smith, G.L.; Cooper, J.E. Clouds and the Earth's Radiant Energy System (CERES): An Earth Observing System Experiment. Bull. Am. Meteorol. Soc. 1996, 77, 853-868. [CrossRef] 
8. Loeb, N.G.; Manalo-Smith, N.; Su, W.; Shankar, M.; Thomas, S. CERES top-of-atmosphere earth radiation budget climate data record: Accounting for in-orbit changes in instrument calibration. Remote Sens. 2016, 8 , 182. [CrossRef]

9. Harries, J.; Crommelynck, D. The geostationary earth radiation budget experiment on MSG-1 and its potential applications. Adv. Space Res. 1999, 24, 915-919. [CrossRef]

10. Harries, J.E.; Russell, J.; Hanafin, J.; Brindley, H.; Futyan, J.; Rufus, J.; Kellock, S.; Matthews, G.; Wrigley, R.; Last, A. The geostationary earth radiation budget project. Bull. Am. Meteorol. Soc. 2005, 86, 945-960. [CrossRef]

11. De Paepe, B.; Ignatov, A.; Dewitte, S.; Ipe, A. Aerosol retrieval over ocean from SEVIRI for the use in GERB Earth's radiation budget analyses. Remote Sens. Environ. 2008, 112, 2455-2468. [CrossRef]

12. Mantziaras, D. EOS Terra Mission Status. In Proceedings of the Earth Science Constellation MOWG Meeting, Goddard Space Flight Center, Greenbelt, MD, USA, 13-15 June 2017.

13. Smith, G.L.; Wong, T.; Bush, K.A. Time-Sampling Errors of Earth Radiation From Satellites: Theory for Outgoing Longwave Radiation. IEEE Trans. Geosci. Remote Sens. 2015, 53, 1656-1665. [CrossRef]

14. Smith, G.L.; Wong, T. Time-Sampling Errors of Earth Radiation from Satellites: Theory for Monthly Mean Albedo. IEEE Trans. Geosci. Remote Sens. 2016, 54, 3107-3115. [CrossRef]

15. Doelling, D.R.; Loeb, N.G.; Keyes, D.F.; Nordeen, M.L.; Morstad, D.; Nguyen, C.; Wielicki, B.A.; Young, D.F.; Sun, M. Geostationary enhanced temporal interpolation for CERES flux products. J. Atmos. Ocean. Technol. 2013, 30, 1072-1090. [CrossRef]

16. Huang, S. Surface temperatures at the nearside of the Moon as a record of the radiation budget of Earth's climate system. Adv. Space Res. 2008, 41, 1853-1860. [CrossRef]

17. Pallé, E.; Goode, P.R. The Lunar Terrestrial Observatory: Observing the Earth using photometers on the Moon's surface. Adv. Space Res. 2009, 43, 1083-1089. [CrossRef]

18. Rogalski, A. Next decade in infrared detectors. In Proceedings of the Electro-Optical and Infrared Systems: Technology and Applications XIV, Warsaw, Poland, 9 October 2017.

19. Lohmeyer, W.Q.; Cahoy, K. Space weather radiation effects on geostationary satellite solid-state power amplifiers. Space Weather 2013, 11, 476-488. [CrossRef]

20. Durante, M.; Cucinotta, F.A. Physical basis of radiation protection in space travel. Rev. Modern Phys. 2011, 83, 1245-1281. [CrossRef]

21. Cressler, J.D.; Mantooth, H.A. Extreme Environment Electronics, 1st ed.; CRC Press: Boca Raton, FL, USA, 2012.

22. Chen, T. Digital Camera System Simulator and Applications; Stanford University: Stanford, CA, USA, 2003.

23. Loeb, N.G.; Kato, S.; Loukachine, K.; Manalo-Smith, N. Angular Distribution Models for Top-of-Atmosphere Radiative Flux Estimation from the Clouds and the Earth's Radiant Energy System Instrument on the Terra Satellite. Part I: Methodology. J. Atmos. Ocean. Technol. 2005, 22, 338-351. [CrossRef]

24. Loeb, N.G.; Kato, S.; Loukachine, K.; Manalo-Smith, N.; Doelling, D.R. Angular distribution models for top-of-atmosphere radiative flux estimation from the Clouds and the Earth's Radiant Energy System instrument on the Terra satellite. Part II: Validation. J. Atmos. Ocean. Technol. 2007, 24, 564-584. [CrossRef]

25. Su, W.; Corbett, J.; Eitzen, Z.; Liang, L. Next-generation angular distribution models for top-of-atmosphere radiative flux calculation from CERES instruments: Methodology. Atmos. Meas. Tech. 2015, 7, 8817-8880. [CrossRef]

26. Giorgini, J.D. Status of the JPL horizons ephemeris system. In Proceedings of the IAU General Assembly, Honolulu, HI, USA, 3-14 August 2015.

27. CERES_SYN1deg_Ed4A Data Quality Summary. Available online: https:/ / ceres.larc.nasa.gov/documents / DQ_summaries/CERES_SYN1deg_Ed4A_DQS.pdf (accessed on 10 January 2018).

28. Smith, G.; Priestley, K.; Loeb, N.; Wielicki, B.; Charlock, T.; Minnis, P.; Doelling, D.; Rutan, D. Clouds and Earth Radiant Energy System (CERES), a review: Past, present and future. Adv. Space Res. 2011, 48, 254-263. [CrossRef]

29. Doelling, D.R.; Sun, M.; Nguyen, L.T.; Nordeen, M.L.; Haney, C.O.; Keyes, D.F.; Mlynczak, P.E. Advances in geostationary-derived longwave fluxes for the CERES synoptic (SYN1deg) product. J. Atmos. Ocean. Technol. 2016, 33, 503-521. [CrossRef]

30. CERES Surface Type ID. Available online: https://ceres.larc.nasa.gov/science_information.php?page= CeresSurfID\# (accessed on 20 September 2017). 
31. CERES EBAF TOA Ed4.0 Data Ordering. Available online: https:/ / ceres-tool.larc.nasa.gov/ord-tool/jsp / EBAF4Selection.jsp (accessed on 15 February 2018).

32. Duan, W.; Huang, S.; Nie, C. Conceptual design of a Moon-based Earth radiation observatory. Int. J. Remote Sens. 2018, 39, 5834-5849. [CrossRef]

33. CERES Algorithm Theoretical Basis Document. Available online: https:/ / ceres.larc.nasa.gov/documents / $\mathrm{ATBD} / \mathrm{pdf} / \mathrm{r2}$ _2/ceres-atbd2.2-s4.4.pdf (accessed on 10 November 2017). 